\title{
Development and validation of a risk prediction model and scoring system for post-endoscopic retrograde cholangiopancreatography pancreatitis
}

\author{
Ruhua Zheng ${ }^{1 \#}$, Mengjie Chen ${ }^{1 \#}$, Xuying Wang ${ }^{3}$, Bingqiang Li $^{3}$, Ting $\mathrm{He}^{3}$, Lei Wang ${ }^{1}$, Guifang $\mathrm{Xu}^{1}$, \\ Yuling Yao ${ }^{1}$, Jun Cao ${ }^{1}$, Yonghua Shen ${ }^{1}$, Yi Wang ${ }^{1}$, Hao Zhu ${ }^{1}$, Bin Zhang ${ }^{1}$, Han Wu ${ }^{1}$, Xiaoping Zou ${ }^{1,2}$, \\ Guoping $\mathrm{He}^{3}$
}

${ }^{1}$ Department of Gastroenterology, Nanjing Drum Tower Hospital Clinical College of Nanjing Medical University, Nanjing, China; ${ }^{2}$ Department of Gastroenterology, The Affiliated Drum Tower Hospital of Nanjing University Medical School, Nanjing, China; ${ }^{3}$ Department of Artificial intelligence, Ewell Technology Enterprise Institute, Hangzhou, China

Contributions: (I) Conception and design: X Zou, L Wang, G Xu; (II) Administrative support: X Zou, G He; (III) Provision of study materials or patients: R Zheng, M Chen; (IV) Collection and assembly of data: Y Yao, J Cao, Y Shen, Y Wang, H Zhu, B Zhang, H Wu; (V) Data analysis and interpretation: X Wang, B Li, T He; (VI) Manuscript writing: R Zheng, M Chen; (VII) Final approval of manuscript: All authors.

\#These authors contributed equally to this work.

Correspondence to: Guoping He. Department of Artificial Intelligence, Ewell Technology Enterprise Institute, No. 66 Dongxin Avenue, Hangzhou 310053, China. Email: hgp@ewell.cc; Xiaoping Zou. Department of Gastroenterology, Nanjing Drum Tower Hospital Clinical College of Nanjing Medical University, No. 321 Zhongshan Road, Nanjing 210008, China. Email: 13770771661@163.com.

Background: A few models have been proposed for the prediction of the risk of post-endoscopic retrograde cholangiopancreatography pancreatitis (PEP), but many include factors that are not assessed routinely. Herein, we intend to develop and validate a predictive model for the occurrence of PEP.

Methods: Data of patients who underwent endoscopic retrograde cholangiopancreatography (ERCP) from January 01, 2016 to May 16, 2019 (training set and internal test set) and from May 17, 2019 to December 25, 2019 (external test set) were retrospectively collected. The performance of the model was validated in the two validation cohorts.

Results: A total of 342 patients were included for the external test set, and 47 (13.7\%) developed PEP. The variables included in the scoring system were gastrectomy history, high direct bilirubin (DBIL), high albumin (ALB), villous type of papillary orifice, nodular type of papillary orifice, pancreatic guidewire passages, precut sphincterotomy, and high operator experience. A total score $>5$ indicated high risk. In the external test set, the area under the curve (AUC) was 0.718 , the sensitivity was 0.723 , and the specificity was 0.676 . In the external test set, the probability of PEP was $6.1 \%, 17.0 \%$, and $37.5 \%$ in patients with low $(<0)$, moderate $(0-5)$, and high (>5) risk scores, respectively.

Conclusions: This study established a scoring system for predicting the risk of PEP using routinely measured clinical variables. Its application in routine work warrants further investigation.

Keywords: Cholangiopancreatography; endoscopic retrograde; pancreatitis; risk factors; models; statistical

Submitted Jul 16, 2020. Accepted for publication Oct 10, 2020.

doi: $10.21037 / \mathrm{atm}-20-5769$

View this article at: http://dx.doi.org/10.21037/atm-20-5769 


\section{Introduction}

Endoscopic retrograde cholangiopancreatography (ERCP) is a procedure extensively used in the diagnosis and treatment of pancreatobiliary diseases, although it does entail some risk $(1,2)$. Infection $(0.6-5 \%)$, hemorrhage $(0.3-2 \%)$, and perforation $(0.1-1.1 \%)$ are occasional complications of this treatment, but the most common complication is acute pancreatitis, which occurs in $1.3-11 \%$ of cases. PostERCP pancreatitis (PEP) is not only the most common complication but also the most feared because of the risk of morbidity and mortality (0.6-1.1\%) (3-5). There is still uncertainty about which patients will develop PEP, and a number of chemical, hydrostatic, enzymatic, mechanical, and thermal factors have been speculated to be involved in PEP. Studies found that any manipulation causing edema of papilla and injury of pancreatic duct may result in PEP. Mechanical injury caused by difficult cannulation and thermal injury caused by using electrocautery current during sphincterotomy lead to edema of pancreatic orifice, obstructing outflow of pancreatic juice, thus may induce PEP. Contrast injection into pancreatic duct and mechanical manipulation in the pancreatic duct may cause activation of protease and eventually result in PEP (3,6-11). Patients at high-risk of PEP should be monitored more closely in order to receive timely preventive treatment (12). Because of the high occurrence and high morbidity of PEP (3), there is an urgent need for reliable models that can predict PEP.

Most of the related studies have examined the influence of different risk factors on the occurrence of PEP, and some have established a prediction model for PEP by combining the previous studies of relevant risk factors (13-15). Many of the models have low credibility due to using too few cases or having a lack of validation, while others are too complex to be applied in clinical practice. Thus, a large number of cases were included in this study, and an external test was conducted. A scoring standard for the prediction of PEP was established to facilitate its use in clinical practice (12).

Therefore, the aim of the present study was to develop and validate a predictive model and scoring system for the occurrence of PEP. We present the following article in accordance with the TRIPOD reporting checklist (available at http://dx.doi.org/10.21037/atm-20-5769).

\section{Methods}

\section{Study design and patients}

For the training and internal test set, patients who underwent ERCP from January 1, 2016, to May 16, 2019, were retrospectively enrolled at Nanjing Drum Tower Hospital Clinical College of Nanjing Medical University. For the external test set, patients were retrospectively enrolled from May 17, 2019, to December 25, 2019, at Nanjing Drum Tower Hospital Clinical College of Nanjing Medical University. The study was conducted in accordance with the Declaration of Helsinki (as revised in 2013). The Medical Ethics Committee of Nanjing Drum Tower Hospital Clinical College of Nanjing Medical University approved this retrospective data-only study (No. 2019135-01). Written informed consent was obtained from all patients.

All patients who underwent ERCP from January 1, 2016, to May 16, 2019, were included. The exclusion criteria were the following: (I) non-native papilla (including postsphincterotomy, post-papillectomy, post-papillary balloon dilatation, and post-choledochojejunostomy); (II) failed operation; (III) age <18 years; (IV) body weight < $40 \mathrm{~kg}$; (V) unable to provide informed consent; (VI) missing any of the indicators (Table S1). The exclusion criteria for the external test set (from May 17, 2019 to December 25, 2019) were the same as those of the training and internal test sets.

\section{Data collection}

For the training and internal test sets, patient-related data and procedure-related data were extracted from the medical charts, based on a review of the risk factors of PEP (3).

Patient-related data included sex, age, surgical history, gastrectomy history, cholecystectomy history, drinking history, smoking history, hypertension, diabetes, coronary heart disease, chronic pancreatitis history, acute pancreatitis history, C-reactive protein (CRP), international normalized ratio (INR), prothrombin time (PT), activated partial thromboplastin time (APTT), $\gamma$-glutamyl transferase (GGT), alanine aminotransferase (ALT), lactate dehydrogenase (LDH), aspartate aminotransferase (AST), uric acid (UA), total cholesterol (TC), total bile acids (TBA), total proteins (TP), triglycerides (TG), albumin (ALB), direct bilirubin (DBIL), alkaline phosphatase (ALP), creatinine (CREA), glucose (GLU), calcium (Ca), sodium $(\mathrm{Na})$, potassium $(\mathrm{K})$, pancreatic diseases, sphincter of Oddi dysfunction (SOD), hilar bile duct stricture, distal biliary stricture, common bile duct stone, and adenoma of the duodenal papilla.

Procedure-related data included precut sphincterotomy, endoscopic sphincterotomy, pancreatic guidewire passages, 
biliary stent placement, papillary balloon dilatation, nasobiliary drainage, difficult biliary cannulation (duration of cannulation attempts $>10$ minutes, and/or $>5$ attempts), contrast injection to the pancreatic duct, epinephrine injection around the papilla, dilated extrahepatic bile duct, type of papillary orifice, pancreatic duct stenting, and operator experience (high grade: $>200$ ERCP procedures total and/or $>50 /$ year).

For the external test set, preoperative data of blood routine, coagulation, biochemistry, and other related laboratory examinations, along with imaging examinations, the process of the ERCP operation, and operator experience were also extracted from the charts. The patient routinely received indomethacin suppository as per the judgment of the operator. If the patient had new or aggravated epigastric pain within 72 hours, abdominal imaging was routinely performed according to the general situation of the patient, and, if necessary, enzyme inhibition treatment was given.

\section{Definitions}

The diagnosis of postoperative pancreatitis was based on the Atlanta criteria (16). At least two of the following elements had to be present: (I) upper abdominal pain suggestive of pancreatitis, usually epigastric pain radiating to the back; (II) serum amylase at least three times the upper limit of normal levels; and (III) computed tomography (CT), magnetic resonance imaging (MRI), or abdominal ultrasound scans suggestive of pancreatitis. In this study, the patients who experienced new-onset or aggravation of epigastric pain within 3 days after ERCP procedure met the diagnostic criteria for PEP. In this study, the serum amylase of each patient was tested at 3,12, and 24 hours after operation. If the amylase levels were 3 times higher than the upper limit of the normal value (the upper limit of the normal value of serum amylase in this hospital was $110 \mathrm{U} / \mathrm{L}$ ), the patient was potentially diagnosed with PEP.

\section{Statistical analysis}

Because the data of training set and internal test set were extremely unbalanced (PEP:No PEP =1:12.8), we used the upsampling method to randomly divide patients who underwent ERCP from January 1, 2016, to May 16, 2019 into a training set and an internal test set in a ratio of 7:3. Stratified sampling was used to ensure the data distribution of the training set and the internal test set were consistent, and then upsampling and 10-fold cross-validation were performed in the training set; that is, the training set was divided into 10 mutually exclusive subsets of similar size, and each subset was kept as consistent as possible in the data distribution, which was obtained by stratified sampling from the training set. Then, each time the positive and negative samples in the union of 9 subsets were upsampled at a ratio of 1:4 as the training set, the remaining subset was used as the internal test set to select the best model. The continuous data were dichotomized according to the cutoff value of each indicator based on the optimal sensitivity and specificity (17). Categorical data were expressed as numbers, rates, and percentages, and were compared with the Chisquare test or Fisher's exact test. In the training set, the relevant factors determined by univariate analysis $(\mathrm{P}<0.15)$ were included in the multivariate logistic regression analysis (backward stepwise regression selection). A receiver operating characteristics (ROC) curve was used to examine the sensitivity and specificity of the model, and the area under the ROC curve (AUC) was used for discrimination. A score was attributed to each variable based on the $\beta$ value of the variables. The total score was then associated with the occurrence of PEP. The resulting model was tested in the training set and the internal test set. Finally, the model was validated using the external test set. All data were analyzed using $\mathrm{R}$ 3.5.2. $\mathrm{P}$ values $<0.05$ were considered statistically significant.

\section{Results}

\section{Characteristics of the patients}

Data of 3,987 patients who underwent ERCP from January 2016 to May 2019 were retrospectively collected. After excluding the patients with non-native papilla, failed ERCP, and unavailable operation records, a total of 2,547 patients were placed into the next phase for the extraction of the indicators. Among these 2,547 patients, 504 patients had missing indicators (Table S1), and so 2,043 patients were ultimately included in the final analysis. From the 2,043 included patients, $30 \%$ were randomly selected to be in the internal test set, and the remaining $70 \%$ were placed in the training set (Figure 1). The frequency of PEP occurrence among the 2,043 included patients was 148 (7.2\%). Table 1 presents the characteristics of the patients. A total of 506 patients who were scheduled to undergo ERCP were retrospectively enrolled from May 17, 2019 to December 25, 2019. Among these, 164 patients were excluded for having non-native papilla. In the end, 342 patients were 


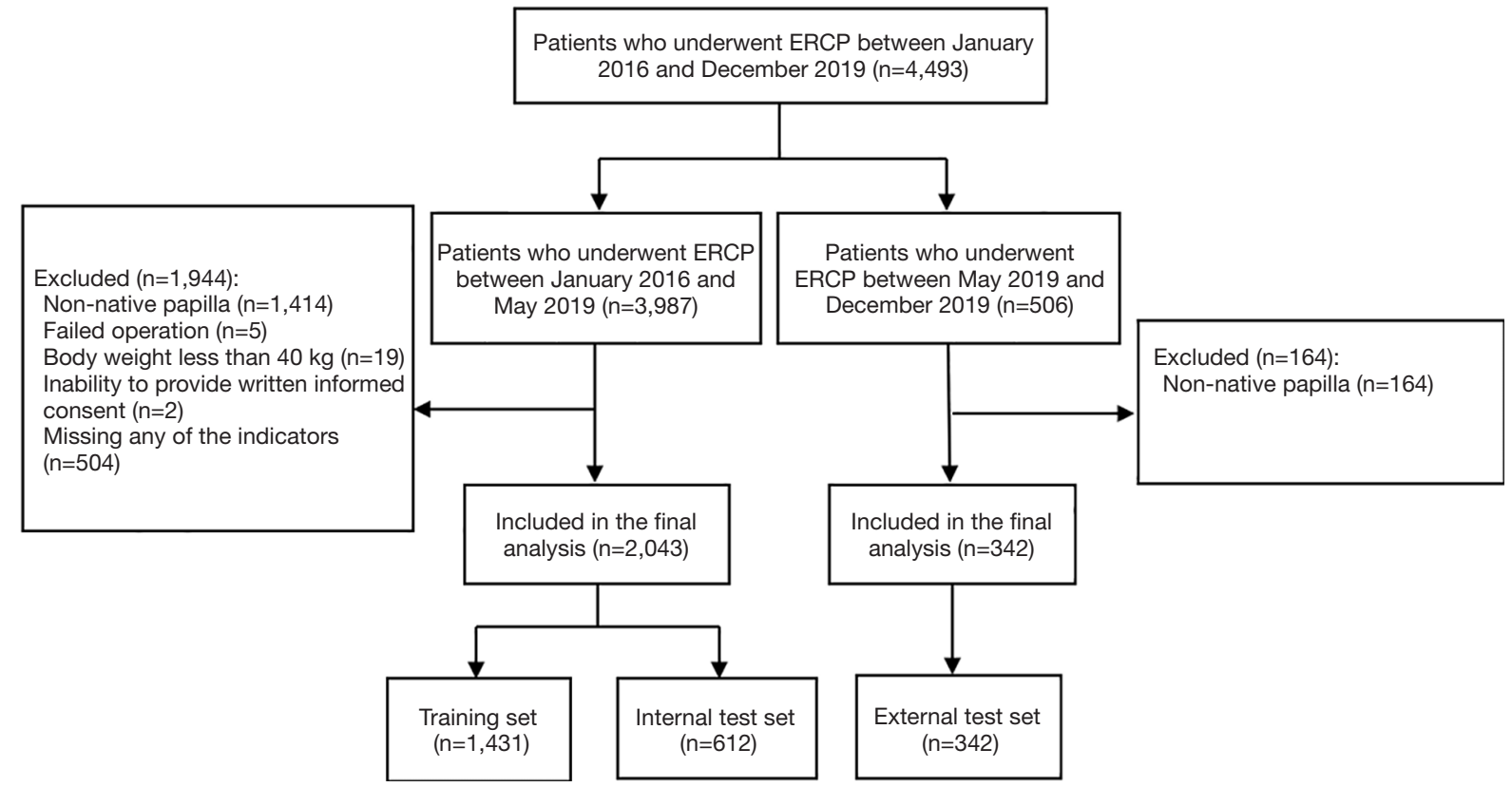

Figure 1 Patient flow diagram showing training set, internal test set, and external test set of patients who underwent ERCP. ERCP, endoscopic retrograde cholangiopancreatography.

included for model validation, and 47 (13.7\%) had PEP.

\section{Univariate and multivariate analysis of the factors associated with PEP}

Univariate analysis was performed using the training set to determine the factors associated with PEP (Table S2), and the identified factors were entered into the multivariate model (Table 2). Gastrectomy history ( $\mathrm{OR}=3.136,95 \%$ CI: $1.843-5.335, \mathrm{P}<0.001$ ), DBIL (OR $=0.557,95 \% \mathrm{CI}$ : $0.430-0.722, \mathrm{P}<0.001)$, ALB (OR $=1.358,95 \%$ CI: 1.048 $1.759, \mathrm{P}=0.021)$, common bile duct stone $(\mathrm{OR}=0.738,95 \%$ CI: $0.562-0.967, \mathrm{P}=0.027$ ), villous type of papillary orifice ( $\mathrm{OR}=2.119,95 \%$ CI: $1.514-3.196, \mathrm{P}<0.001)$, nodular type of papillary orifice $(\mathrm{OR}=4.477,95 \% \mathrm{CI}: 1.370-2.784$, $\mathrm{P}<0.001)$, precut sphincterotomy $(\mathrm{OR}=1.953,95 \% \mathrm{CI}$ : 1.370-2.784, $\mathrm{P}<0.001$ ), and high operator experience (OR $=0.691,95 \%$ CI: $0.507-0.941, \mathrm{P}=0.019)$ were independently associated with the risk of PEP (Figure 2). Among the above-mentioned variables, high DBIL, common bile duct stone, and high operator experience were protective factors, while the other variables were risk factors. A predictive model was then constructed as follows: Logit $(\mathrm{P}=\mathrm{PEP})$ $=-2.27+1.14 \times$ gastrectomy history $-0.58 \times$ DBIL +0.31 $\times \mathrm{ALB}+0.67 \times$ precut sphincterotomy $+0.81 \times$ pancreatic guidewire passages $-0.37 \times$ high operator experience -0.30 $\times$ common bile duct stone $+1.50 \times$ nodular type of papillary orifice (or $0.79 \times$ villous type of papillary orifice). Scores were attributed to each variable (Table 3).

\section{ROC analysis}

The model in the training set had an AUC of 0.793, a sensitivity of 0.727 , and a specificity of 0.797 ; the model in the internal test set had an AUC of 0.725 , a sensitivity of 0.705 , and a specificity of 0.700 ; the model in the external test set had an AUC of 0.718 , a sensitivity of 0.723 , and a specificity of 0.676 (Figure 3).

\section{Predictive ability of the model}

The variables included in the scoring system were gastrectomy history (score $=5.5$ ), high DBIL (DBIL $>7.4 \mu \mathrm{mol} / \mathrm{L}$; score $=-3)$, high ALB (ALB >37.6 g/L; score $=1.5$ ), common bile duct stone ( score $=-1.5$ ), villous type of papillary orifice (score $=4$ ), nodular type of papillary orifice (score $=7.5)$, pancreatic guidewire passages $($ score $=4$ ), precut sphincterotomy (score $=3$ ), and high operator experience (score $=-2$ ) (Table 2). Scores of 0 and 5 were determined as the optimal cutoff points for low risk (score $\leq 0)$ and 
Table 1 Baseline characteristics in the training and test set

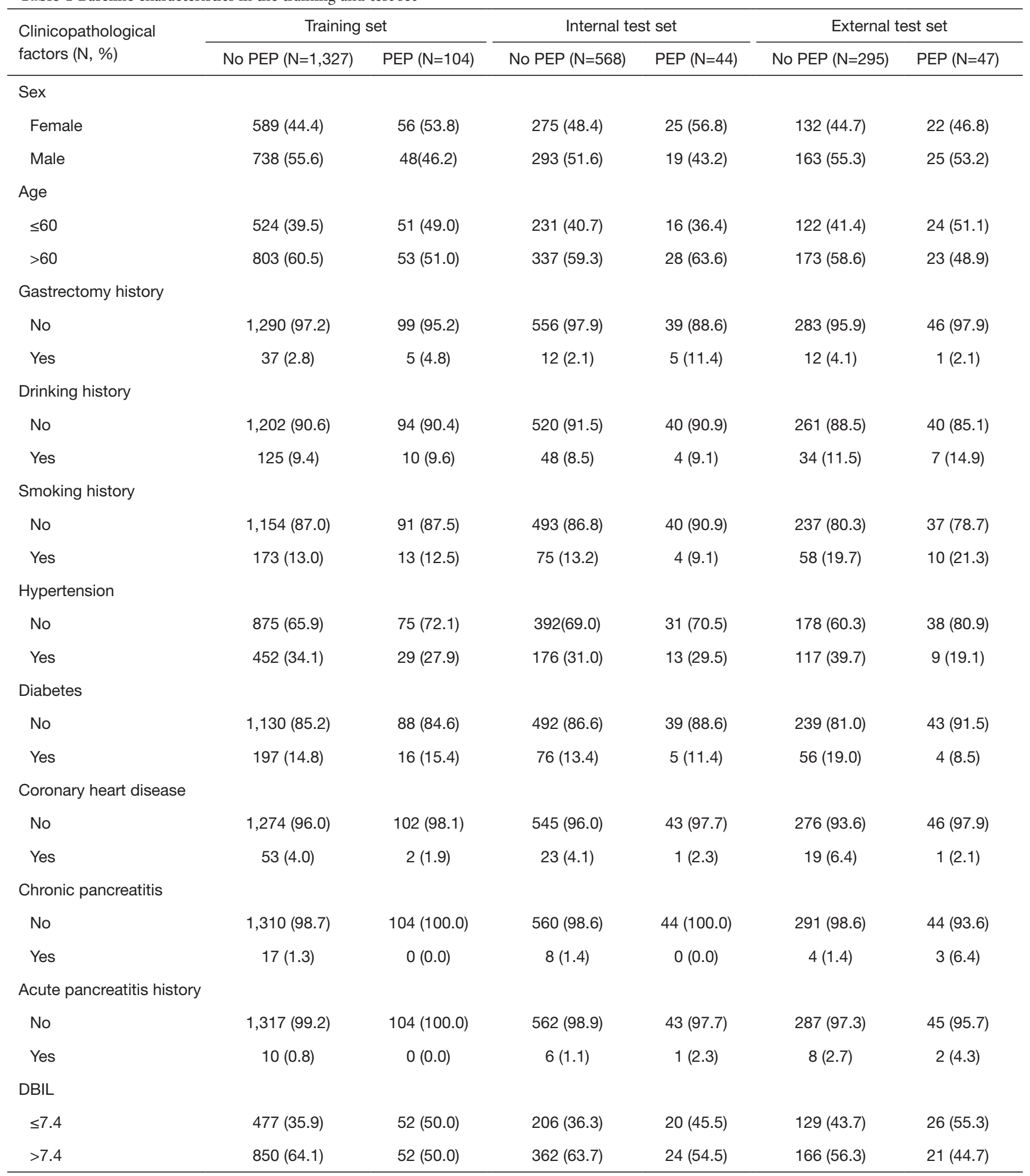

Table 1 (continued) 
Table 1 (continued)

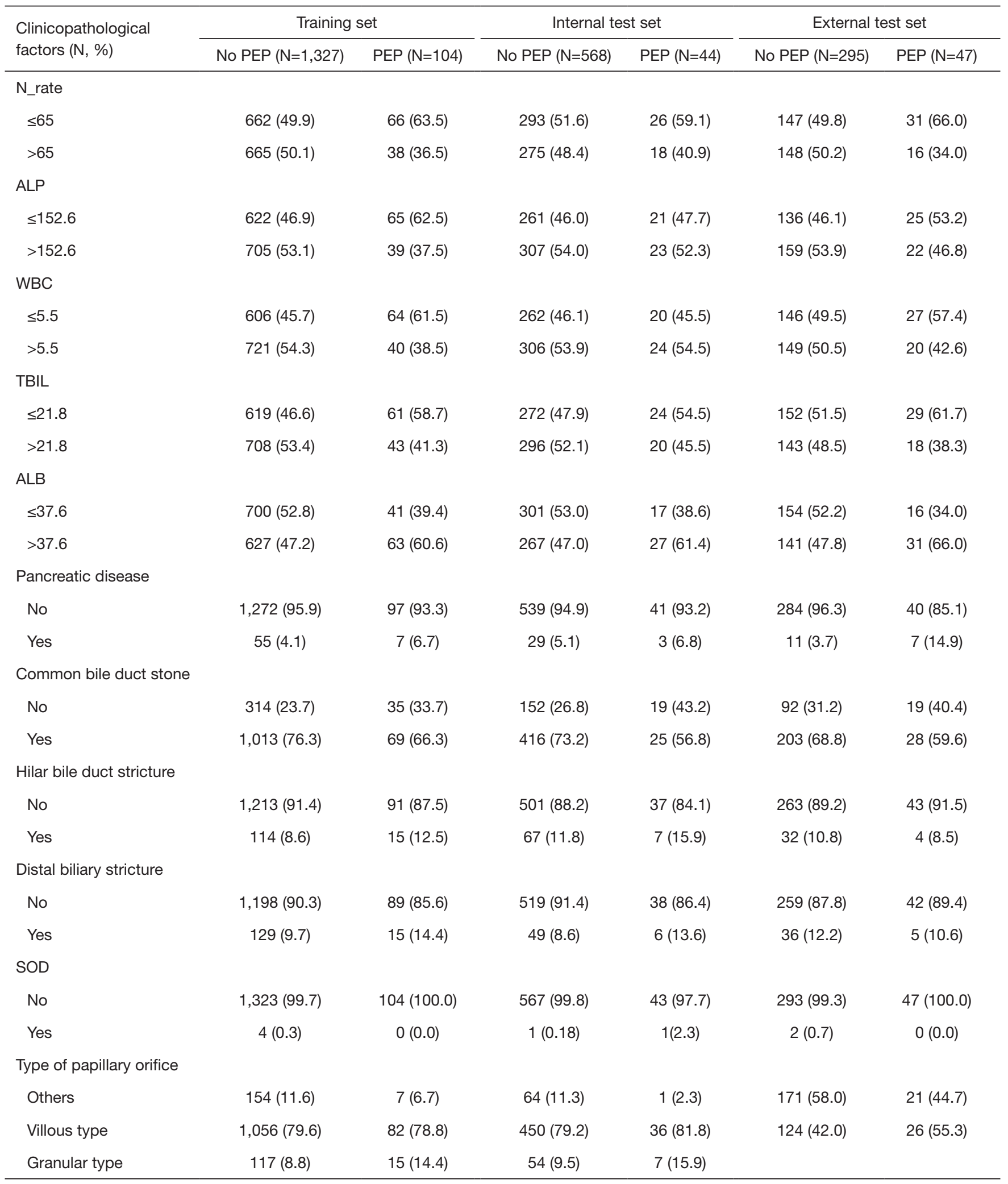

Table 1 (continued) 
Table 1 (continued)

\begin{tabular}{|c|c|c|c|c|c|c|}
\hline $\begin{array}{l}\text { Clinicopathological } \\
\text { factors }(\mathrm{N}, \%)\end{array}$ & \multicolumn{2}{|c|}{ Training set } & \multicolumn{2}{|c|}{ Internal test set } & \multicolumn{2}{|c|}{ External test set } \\
\hline \multicolumn{7}{|c|}{ Precut sphincterotomy } \\
\hline No & $1,230(92.7)$ & $87(83.7)$ & $522(91.9)$ & $40(90.9)$ & 275 (93.2) & $40(85.1)$ \\
\hline Yes & $97(7.3)$ & $17(16.3)$ & $46(8.1)$ & $4(9.1)$ & $20(6.8)$ & 7 (14.9) \\
\hline No & $344(25.9)$ & $27(26.0)$ & $147(25.9)$ & $12(27.3)$ & $66(22.4)$ & $8(17.0)$ \\
\hline Yes & $983(74.1)$ & $77(74.0)$ & $421(74.1)$ & $32(72.7)$ & $229(77.6)$ & $39(83.0)$ \\
\hline \multicolumn{7}{|c|}{ Pancreatic guidewire passages } \\
\hline No & $1,006(75.8)$ & $56(53.8)$ & $418(73.6)$ & $21(47.7)$ & $178(60.3)$ & $15(31.9)$ \\
\hline \multicolumn{7}{|c|}{ Biliary stent placement } \\
\hline No & $978(73.7)$ & $67(64.4)$ & $413(72.7)$ & $22(50)$ & $250(84.7)$ & $42(89.4)$ \\
\hline Yes & $349(26.3)$ & $37(35.6)$ & $155(27.3)$ & $22(50)$ & $45(15.3)$ & $5(10.6)$ \\
\hline \multicolumn{7}{|c|}{ Papillary balloon dilation } \\
\hline No & $933(70.3)$ & $77(74.0)$ & $427(75.2)$ & $30(68.2)$ & $193(65.4)$ & $32(68.1)$ \\
\hline Yes & $394(29.7)$ & $26(26.0)$ & $141(24.8)$ & $14(31.8)$ & $102(34.6)$ & $15(31.9)$ \\
\hline \multicolumn{7}{|l|}{ Nasobiliary drainage } \\
\hline No & $306(23.1)$ & $31(29.8)$ & $124(21.8)$ & $14(31.8)$ & $68(23.1)$ & $18(38.3)$ \\
\hline Yes & 1,021 (76.9) & $73(70.2)$ & 444 (78.2) & $30(68.2)$ & 227 (76.9) & $29(61.7)$ \\
\hline No & 1,185 (89.3) & $88(84.6)$ & $518(91.2)$ & $39(88.6)$ & $254(86.1)$ & $32(68.1)$ \\
\hline Yes & $142(10.7)$ & $16(15.4)$ & $50(8.8)$ & $5(11.4)$ & $41(13.9)$ & 15 (31.9) \\
\hline \multicolumn{7}{|l|}{ Cholangiectasis } \\
\hline No & $634(47.8)$ & $60(57.7)$ & $279(49.1)$ & $28(63.6)$ & $62(21.0)$ & $13(27.7)$ \\
\hline Yes & 693 (52.2) & $44(42.3)$ & $289(50.9)$ & $16(36.4)$ & $233(79.0)$ & $34(72.3)$ \\
\hline \multicolumn{7}{|c|}{ Pancreatic duct stenting } \\
\hline No & 1,047 (78.9) & $63(60.6)$ & $434(76.4)$ & $24(54.5)$ & $212(71.9)$ & $22(46.8)$ \\
\hline Yes & $280(21.1)$ & $41(39.4)$ & $134(23.6)$ & $20(45.5)$ & $83(28.1)$ & $25(53.2)$ \\
\hline \multicolumn{7}{|l|}{ Operator experience } \\
\hline Low & $182(13.7)$ & $18(17.3)$ & $72(12.7)$ & $4(9.1)$ & $136(46.1)$ & $25(53.2)$ \\
\hline High & $1,145(86.3)$ & $86(82.7)$ & $496(87.3)$ & 40 (90.9) & $159(53.9)$ & $22(46.8)$ \\
\hline
\end{tabular}

PEP, post-ERCP pancreatitis; DBIL, direct bilirubin; ALP, alkaline phosphatase; WBC, white blood cells; TBIL, total bilirubin; ALB, albumin; SOD, sphincter of Oddi dysfunction. 
Table 2 Multivariate regression model

\begin{tabular}{|c|c|c|c|c|c|c|}
\hline Variables & OR & $95 \% \mathrm{Cl}$ & $\beta$ & $P$ & Cutoff value & Scoring \\
\hline Gastrectomy history & 3.136 & $1.843-5.335$ & 1.14 & $<0.001$ & & 5.5 \\
\hline DBIL & 0.557 & $0.430-0.722$ & -0.58 & $<0.001$ & $>7.4$ & -3 \\
\hline ALB & 1.358 & $1.048-1.759$ & 0.31 & 0.021 & $>37.6$ & 1.5 \\
\hline \multicolumn{7}{|l|}{ Procedure-related } \\
\hline Papillary orifice (villous type) & 2.119 & $1.514-3.196$ & 0.79 & $<0.001$ & & 4 \\
\hline Papillary orifice (Nodular type) & 4.477 & $2.755-7.275$ & 1.50 & $<0.001$ & & 7.5 \\
\hline Pancreatic guidewire passages & 2.245 & $1.743-2.891$ & 0.81 & $<0.001$ & & 4 \\
\hline High operator experience & 0.691 & $0.507-0.941$ & -0.37 & 0.019 & & -2 \\
\hline
\end{tabular}

OR, odds ratio; $\mathrm{Cl}$, confidence interval; DBIL, direct bilirubin; $\mathrm{ALB}$, albumin.

\begin{tabular}{|c|c|c|c|c|c|}
\hline FACTORS & OR & $95 \%$ CIs & & & $P$ \\
\hline Gastrectomy history 1 & 3.136 & $(1.843,5.335)$ & & $\longmapsto$ & $2.5 \mathrm{e}-05$ \\
\hline DBIL 1 & 0.5571 & $(0.43,0.7219)$ & $\longmapsto-1$ & & $9.625 \mathrm{e}-06$ \\
\hline ALB 1 & 1.358 & $(1.048,1.759)$ & & $\longmapsto-1$ & 0.02083 \\
\hline precut sphincterotomy 1 & 1.953 & $(1.37,2.784)$ & & $\longmapsto-1$ & 0.0002177 \\
\hline Pancreatic guidewire passages 1 & 2.245 & $(1.743,2.891)$ & & $\longmapsto-1$ & $3.807 \mathrm{e}-10$ \\
\hline Operator experience 1 & 0.6914 & $(0.5078,0.9414)$ & $\longmapsto$ & & 0.0191 \\
\hline Common bile duct stone 1 & 0.7379 & $(0.5629,0.9672)$ & $\longmapsto$ & & 0.0277 \\
\hline nodular type of papillary orifice & 4.477 & $(2.755,7.275)$ & & $\mapsto$ & $1.429 \mathrm{e}-09$ \\
\hline Villus type of papillary orifice & 2.199 & $(1.514,3.196)$ & & $\longmapsto$ & $3.561 \mathrm{e}-05$ \\
\hline
\end{tabular}

Figure 2 Forrest plot for the factors involved in the risk of post-ERCP pancreatitis. ERCP, endoscopic retrograde cholangiopancreatography.

high risk (score $>5$ ), with moderate risk located in between (score between 1 and 5). In the training set, compared with the low-risk group, the occurrence of PEP was increased in the moderate-risk $(\mathrm{OR}=2.87,95 \% \mathrm{CI}: 1.85-4.48$, $\mathrm{P}<0.0001)$ and high-risk $(\mathrm{OR}=4.52,95 \% \mathrm{CI}: 2.43-8.15$, $\mathrm{P}<0.0001)$ groups. In the internal test set, the moderaterisk $(\mathrm{OR}=4.58,95 \% \mathrm{CI}: 2.23-9.97, \mathrm{P}<0.0001)$ and highrisk $(\mathrm{OR}=9.51,95 \% \mathrm{CI}: 3.72-24.14, \mathrm{P}<0.0001)$ groups had an increased occurrence of PEP compared with the lowrisk group. In the external test set, compared with the low- risk group, the occurrence of $\mathrm{PEP}$ was also increased in the moderate-risk $(\mathrm{OR}=3.13,95 \% \mathrm{CI}: 1.48-7.24, \mathrm{P}<0.0001)$ and high-risk $(\mathrm{OR}=9.20,95 \% \mathrm{CI}: 3.15-27.29, \mathrm{P}<0.0001)$ groups (Table 3).

\section{Probability of PEP}

Figure 4 presents the probability of PEP. The probability of PEP correlated with the degree of risk in the groups. In the training set, among patients at low, moderate, and 
Table 3 Scoring system table

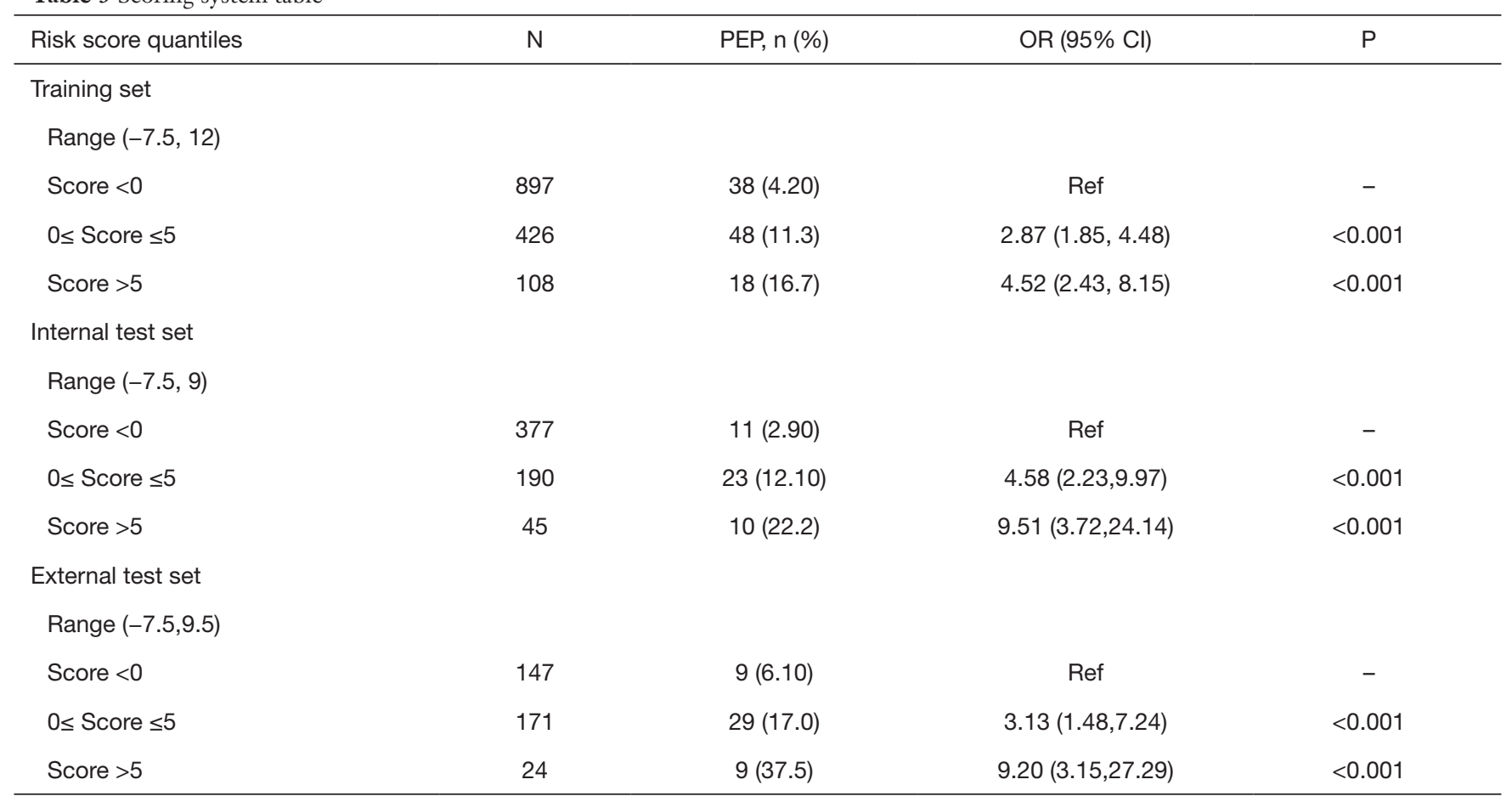

PEP, post-ERCP pancreatitis; OR, odds ratio; Cl, confidence interval.

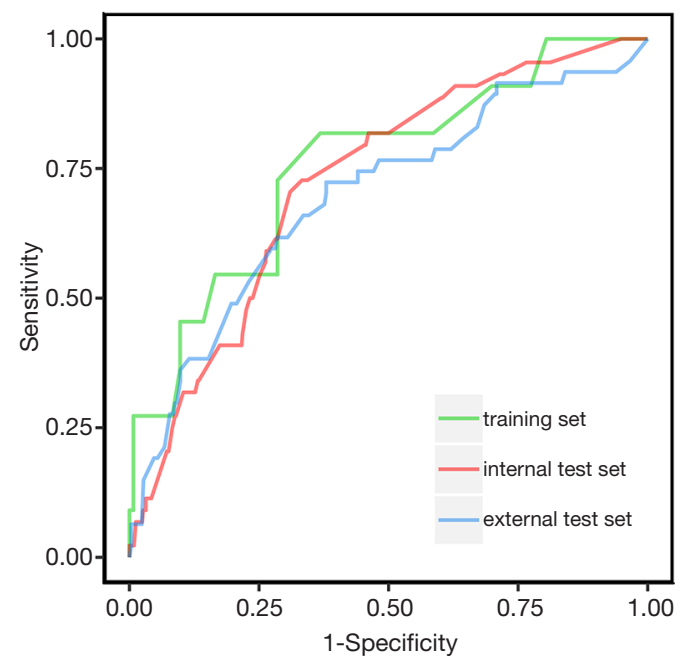

Figure 3 ROC curve for risk of post-ERCP pancreatitis in the training set, internal test set, and external test set. ROC, receiver operating characteristics; ERCP, endoscopic retrograde cholangiopancreatography.

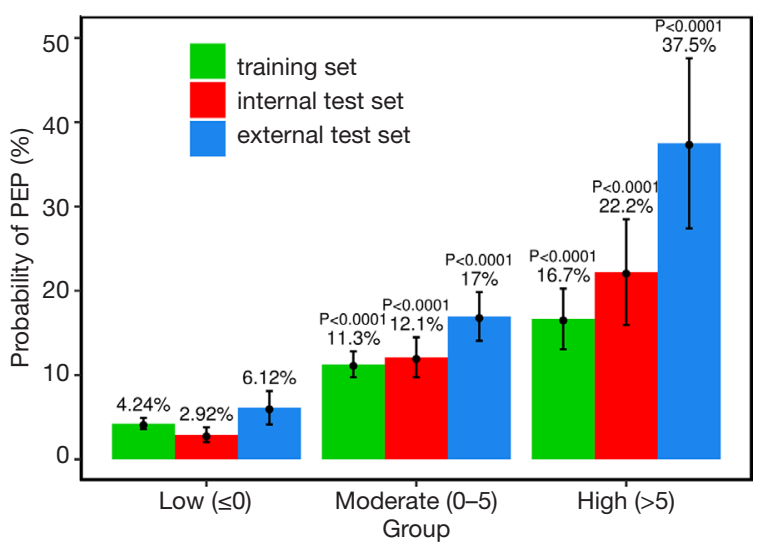

Figure 4 Histogram for the risk of PEP. According to the scoring system, a histogram of the proportions of the low-, medium-, and high-risk populations were drawn. PEP, post-ERCP pancreatitis; ERCP, endoscopic retrograde cholangiopancreatography. 
high risk of PEP, the actual occurrence of PEP was $4.2 \%$, $11.3 \%$, and $16.7 \%$, respectively. In the internal test set, among patients at low, moderate, and high risk of PEP, the actual occurrence of PEP was $2.9 \%, 12.1 \%$, and $22.2 \%$, respectively; in the external test set, those frequencies were $6.1 \%, 17.0 \%$, and $37.5 \%$, respectively.

\section{Discussion}

A few models have been proposed for the prediction of the risk of PEP (13-15). Many of the models have low credibility due to using a low number of cases or from a lack of validation, while others are too complex for clinical practice. Therefore, this study aimed to develop and validate a predictive model and scoring system for the occurrence of PEP using comprehensive patient, operation, and operator variables. This study successfully established a predictive model for PEP using nine variables that yielded an AUC of 0.718 , a sensitivity of 0.723 , and a specificity of 0.676 in the external test set of patients.

The pathogenesis of PEP is complex and is probably the result of the combination of chemical, hydrostatic, enzymatic, mechanical, and thermal insults that occur during ERCP (3). Previous studies identified a number of factors that could be associated with PEP. Two reviews showed that patient-related factors (young age, female sex, possible sphincter of Oddi dysfunction, history of recurrent pancreatitis, history of PEP, and normal TBIL and DBIL), procedure-related factors (ampullectomy, multiple pancreatic duct injections, difficult cannulation, pancreatic sphincterotomy, precut sphincterotomy, balloon dilation, and minor papilla sphincterotomy), and operatorrelated factors (inadequate training, low experience, and trainees involved in the procedure) were associated with an increased risk of PEP $(3,11)$. Iorgulescu et al. (10) showed that difficult sphincterotomy, precut sphincterotomy, failure of deobstruction, pancreatic sphincterotomy, multiple pancreatic duct injections, sphincter of Oddi dysfunction, and the absence of chronic pancreatitis changes were risk factors for PEP. On the basis of the previous studies, this study also analyzed multiple factors according to patientrelated, operation-related, and operator-related categories to determine the factors associated with PEP and construct a prediction model.

The present study identified gastrectomy history, high ALB, villous type of papillary orifice, nodular type of papillary orifice, pancreatic guidewire passages, and precut sphincterotomy as being risk factors for PEP, and high DBIL, common bile duct stone, and high operator experience as being protective factors. Because of the surgically altered anatomy, ERCP is more difficult in patients with previous gastrectomy compared with patients with the native anatomy. The papillary area in the second part of the duodenum can only be reached through the afferent loop. As a result, the papilla of Vater appears upside-down compared with its orientation during standard ERCP, making ECRP more difficult (18). High serum ALB levels might be an indicator of dehydration, and aggressive hydration has been reported as a means to prevent PEP. Previous studies suggest that aggressive hydration with lactated Ringer's solution may alleviate activation of zymogen and inflammation response, and improve perfusion of pancreas parenchyma (19). Our study suggests that high serum ALB levels are a risk factor for PEP, but there is currently no relevant literature suggesting a relationship between ALB and PEP. Thus, further multicenter studies are needed for confirmation, and basic research is required to clarify its underlying mechanism. The type of papillary orifice of Vater will influence the difficulty of cannulation $(20,21)$. A precut sphincterotomy is usually applied after difficult cannulation, which will increase the success rate of ERCP but will increase the risk of PEP (22). Previous studies have found that the guidewire entering the pancreatic duct may be a risk factor for $\operatorname{PEP}(23,24)$, and our research also confirms this. A pancreatic duct guidewire entering too deep may cause a certain degree of damage to the pancreatic duct, thereby promoting the occurrence of PEP (25-27). On the other hand, high DBIL, common bile duct stone, and high operator experience were identified as protective factors for PEP. The experience of the operator is a well-recognized factor in avoiding iatrogenic injury during ERCP and the occurrence of PEP $(3,8)$ Furthermore, one study revealed that patients with obstructive indications of ERCP, such as common bile duct stone and high DBIL levels, tolerated ERCP better than those without such indications (28).

Previous researchers have tried to propose models based on a number of factors for the prediction of the risk of PEP $(8,13,14)$. The major limitations of these models include a low credibility due to too small sample size, a lack of validation, and impracticable complexity. DiMagno et al. (13) constructed two models (pre-ERCP and post-ERCP) based on protective (current smoking, chronic liver diseasebiliary, and chronic liver disease-transplant/hepatectomy complications) and predictive (younger age, possible sphincter of Oddi dysfunction, pancreatic sphincterotomy, 
and moderate-difficult cannulation) factors, and achieved an AUC of 0.73-0.74. Meanwhile, Coté et al. (14) showed that severe comorbidities, high pain, longer procedure, sphincter of Oddi performance, and greater use of opiates and anxiolytics were independently associated with PEP and could be used to construct a model with an AUC of 0.780.83 . Cheng et al. (8) constructed a multivariate risk model based on minor papilla sphincterotomy, possible sphincter of Oddi dysfunction, history of PEP, younger age, multiple contrast injections, and trainee involvement. However, these models are more or less flawed. For example, opioid and anxiolytic use and minor papilla cannulation occur in only a small proportion of the ERCP population, and thus have limited clinical significance. In the present study, gastrectomy history, high DBIL, high ALB, common bile duct stones, villous type of papillary orifice, nodular type of papillary orifice, pancreatic guidewire passages, precut sphincterotomy, and high operator experience were independently associated with PEP and included in the model, which achieved AUCs of 0.718-0.793, a sensitivity of $0.705-0.727$, and a specificity of $0.676-0.797$. Using clinical variables that are routinely assessed/observed in the clinical setting, the model could identify three risk levels with good discrimination among the three groups. Some factors identified in the present study and used to construct the model were also included in the previous models $(8,13,14)$, but not all. Nevertheless, as described above, all included factors had a biological basis as a possible explanation for their association with the occurrence of PEP. Differences among models can be due to a number of factors, including the study population and hospital setting. Additional studies are required to validate the model in a variety of populations and to improve upon its performance.

Some limitations to this study should also be addressed. All patients were from a single-center study, and the sample was relatively small, which might have introduced some bias based on local practice. A multicenter study is required to validate the model and refine its prediction ability. Moreover, a substantial number of patients had to be excluded because of missing data, but only patients with complete datasets were included. Additional studies with larger sample sizes or prospective studies are necessary to reduce these biases.

\section{Conclusions}

This study successfully established and validated a predictive model and scoring system for PEP based on variables that are routinely assessed or observed during the management of patients undergoing ERCP. The model stratified risk into three levels and achieved AUCs of $0.718-0.793$, a sensitivity of $0.705-0.727$, and a specificity of 0.676-0.797. This model may become useful model for the risk prediction of PEP, but further validation in routine practice is necessary.

\section{Acknowledgments}

Funding: The research was supported by the National Natural Science Foundation of China (no. 81871947).

\section{Footnote}

Reporting Checklist: The authors have completed the TRIPOD reporting checklist. Available at http://dx.doi. org/10.21037/atm-20-5769

Data Sharing Statement: Available at http://dx.doi. org/10.21037/atm-20-5769

Conflicts of Interest: All authors have completed the ICMJE uniform disclosure form (available at http://dx.doi. org/10.21037/atm-20-5769). The authors have no conflicts of interest to declare.

Ethical Statement: The authors are accountable for all aspects of the work in ensuring that questions related to the accuracy or integrity of any part of the work are appropriately investigated and resolved. The study was conducted in accordance with the Declaration of Helsinki (as revised in 2013). The Medical Ethics Committee of Nanjing Drum Tower Hospital Clinical College of Nanjing Medical University approved this retrospective data-only study (No. 2019-135-01). Written informed consent was obtained from all patients.

Open Access Statement: This is an Open Access article distributed in accordance with the Creative Commons Attribution-NonCommercial-NoDerivs 4.0 International License (CC BY-NC-ND 4.0), which permits the noncommercial replication and distribution of the article with the strict proviso that no changes or edits are made and the original work is properly cited (including links to both the formal publication through the relevant DOI and the license). See: https://creativecommons.org/licenses/by-ncnd/4.0/. 


\section{References}

1. Costi R, Gnocchi A, Di Mario F, et al. Diagnosis and management of choledocholithiasis in the golden age of imaging, endoscopy and laparoscopy. World J Gastroenterol 2014;20:13382-401.

2. Katzarov AK, Dunkov ZI, Popadiin I, et al. How to measure quality in endoscopic retrograde cholangiopancreatography (ERCP). Ann Transl Med 2018;6:265.

3. Badalov N, Tenner S, Baillie J. The Prevention, recognition and treatment of post-ERCP pancreatitis. JOP 2009;10:88-97.

4. Kochar B, Akshintala VS, Afghani E, et al. Incidence, severity, and mortality of post-ERCP pancreatitis: a systematic review by using randomized, controlled trials. Gastrointest Endosc 2015;81:143-9.e9.

5. Boškoski I, Tringali A, Costamagna G. Teaching endoscopic retrograde cholangiopancreatography cannulation. Transl Gastroenterol Hepatol 2019;4:30.

6. Freeman ML, DiSario JA, Nelson DB, et al. Risk factors for post-ERCP pancreatitis: a prospective, multicenter study. Gastrointest Endosc 2001;54:425-34.

7. Freeman ML, Guda NM. Prevention of post-ERCP pancreatitis: a comprehensive review. Gastrointest Endosc 2004;59:845-64.

8. Cheng CL, Sherman S, Watkins JL, et al. Risk factors for post-ERCP pancreatitis: a prospective multicenter study. Am J Gastroenterol 2006;101:139-47.

9. Mehta SN, Pavone E, Barkun JS, et al. Predictors of post-ERCP complications in patients with suspected choledocholithiasis. Endoscopy 1998;30:457-63.

10. Iorgulescu A, Sandu I, Turcu F, et al. Post-ERCP acute pancreatitis and its risk factors. J Med Life 2013;6:109-13.

11. Parekh PJ, Majithia R, Sikka SK, et al. The "Scope" of Post-ERCP Pancreatitis. Mayo Clin Proc 2017;92:434-48.

12. Morales SJ, Sampath K, Gardner TB. A Review of Prevention of Post-ERCP Pancreatitis. Gastroenterol Hepatol (N Y) 2018;14:286-92.

13. DiMagno MJ, Spaete JP, Ballard DD, et al. Risk models for post-endoscopic retrograde cholangiopancreatography pancreatitis (PEP): smoking and chronic liver disease are predictors of protection against PEP. Pancreas 2013;42:996-1003.

14. Coté GA, Lynch S, Easler JJ, et al. Development and Validation of a Prediction Model for Admission After Endoscopic Retrograde Cholangiopancreatography. Clin Gastroenterol Hepatol 2015;13:2323-32.e329.
15. Sutton VR, Hong MK, Thomas PR. Using the 4-hour Post-ERCP amylase level to predict post-ERCP pancreatitis. JOP 2011;12:372-6.

16. Banks PA, Bollen TL, Dervenis C, et al. Classification of acute pancreatitis--2012: revision of the Atlanta classification and definitions by international consensus. Gut 2013;62:102-11.

17. Budczies J, Klauschen F, Sinn BV, et al. Cutoff Finder: a comprehensive and straightforward Web application enabling rapid biomarker cutoff optimization. PLoS One 2012;7:e51862.

18. Byun JW, Kim JW, Sung SY, et al. Usefulness of Forward-Viewing Endoscope for Endoscopic Retrograde Cholangiopancreatography in Patients with Billroth II Gastrectomy. Clin Endosc 2012;45:397-403.

19. Smeets XJNM, da Costa DW, Fockens P, et al. Fluid hydration to prevent post-ERCP pancreatitis in averageto high-risk patients receiving prophylactic rectal NSAIDs (FLUYT trial): study protocol for a randomized controlled trial. Trials 2018;19:207.

20. Haraldsson E, Lundell L, Swahn F, et al. Endoscopic classification of the papilla of Vater. Results of an interand intraobserver agreement study. United European Gastroenterol J 2017;5:504-10.

21. Testoni PA, Mariani A, Aabakken L, et al. Papillary cannulation and sphincterotomy techniques at ERCP: European Society of Gastrointestinal Endoscopy (ESGE) Clinical Guideline. Endoscopy 2016;48:657-83.

22. Davee T, Garcia JA, Baron TH. Precut sphincterotomy for selective biliary duct cannulation during endoscopic retrograde cholangiopancreatography. Ann Gastroenterol 2012;25:291-302.

23. Tse F, Yuan Y, Moayyedi P, et al. Double-guidewire technique in difficult biliary cannulation for the prevention of post-ERCP pancreatitis: a systematic review and metaanalysis. Endoscopy 2017;49:15-26.

24. Krill JT, DaVee T, Edwards JS, et al. Risk of PostEndoscopic Retrograde Cholangiopancreatography Pancreatitis After Double-Guidewire Biliary Cannulation in an Average-Risk Population. Pancreas 2018;47:748-52.

25. Phillip V, Pukitis A, Epstein A, et al. Pancreatic stenting to prevent post-ERCP pancreatitis: a randomized multicenter trial. Endosc Int Open 2019;7:E860-8.

26. Yin HK, Wu HE, Li QX, et al. Pancreatic Stenting Reduces Post-ERCP Pancreatitis and Biliary Sepsis in High-Risk Patients: A Randomized, Controlled Study. Gastroenterol Res Pract 2016;2016:9687052.

27. Ito K, Fujita N, Kanno A, et al. Risk factors for post- 
ERCP pancreatitis in high risk patients who have undergone prophylactic pancreatic duct stenting: a multicenter retrospective study. Intern Med 2011;50:2927-32.

28. Sahakian AB, Buxbaum JL, Van Dam J. Prevention

Cite this article as: Zheng $\mathrm{R}$, Chen $\mathrm{M}$, Wang $\mathrm{X}$, Li B, He T, Wang L, Xu G, Yao Y, Cao J, Shen Y, Wang Y, Zhu H, Zhang B, Wu H, Zou X, He G. Development and validation of a risk prediction model and scoring system for postendoscopic retrograde cholangiopancreatography pancreatitis. Ann Transl Med 2020;8(20):1299. doi: 10.21037/atm-20-5769 and management of post-ERCP pancreatitis. JOP 2014;15:544-51.

(English Language Editor: J. Gray) 
Supplementary

Table S1 Missing data

\begin{tabular}{lccccc}
\hline Variables & Missing $(\mathrm{n})$ & Variables & Missing $(\mathrm{n})$ & Variables & Missing $(\mathrm{n})$ \\
\hline CRP & 76 & INR & 212 & PT & 212 \\
$\pi T$ & 212 & APTT & 212 & GGT & 44 \\
ALT & 44 & LDH & 35 & AST & 35 \\
UA & 51 & TC & 77 & TBA & 44 \\
TP & 43 & TG & 77 & ALB & CREA \\
DBIL & 44 & ALP & 44 & K & 43 \\
GLU & 51 & Na & 46 & RBC & 51 \\
N_rate & 1 & WBC & 1 & Whether there was postoperative pancreatitis & 56 \\
PLT & 1 & Hb & 1 & & 6 \\
Papillary orifice type & 4 & Ca & 46 & & 56 \\
\hline
\end{tabular}


Table S2 Univariate regression analysis

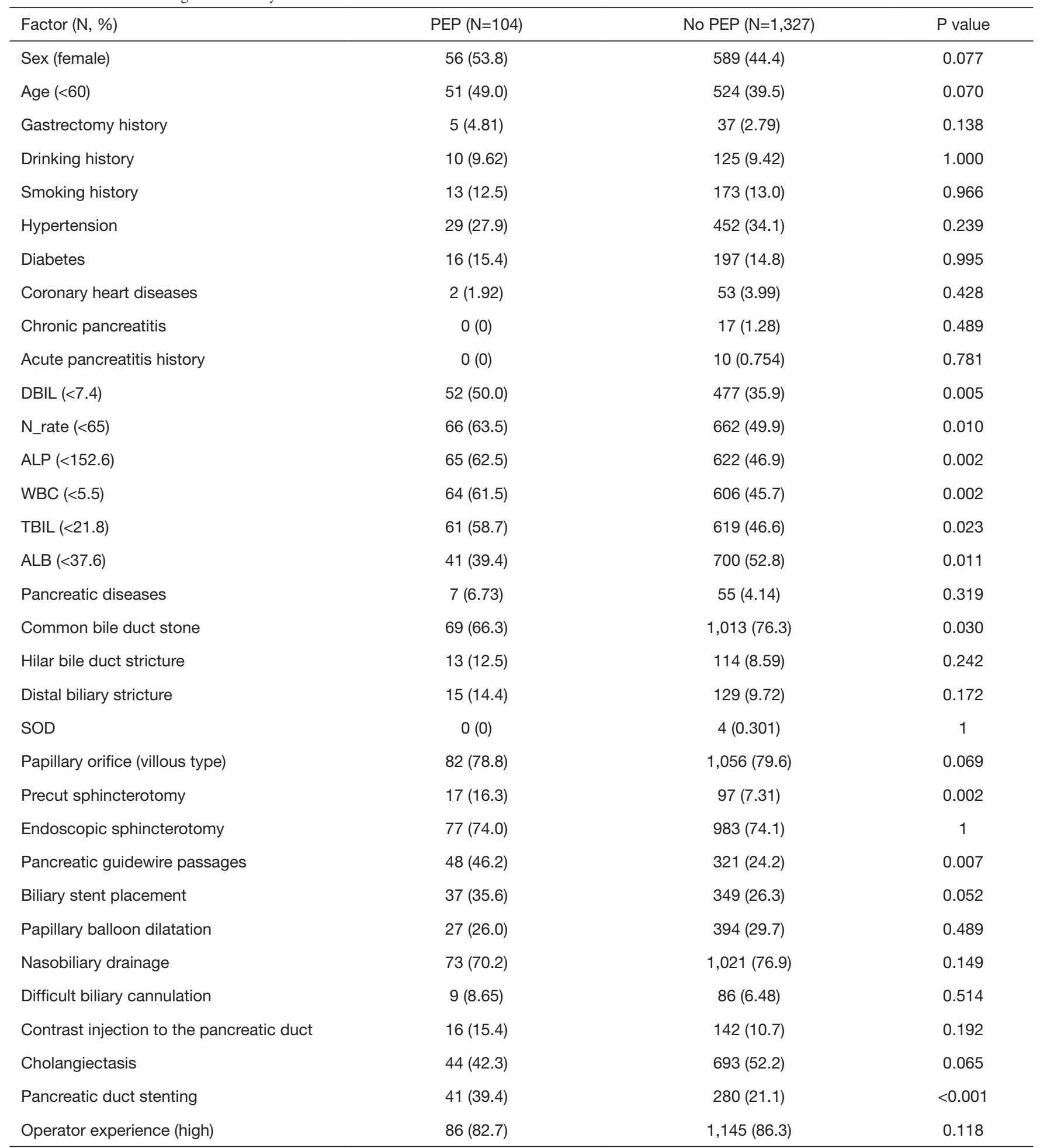

PEP, post-ERCP pancreatitis; DBIL, direct bilirubin; ALP, alkaline phosphatase; WBC, white blood cells; TBIL, total bilirubin; ALB, albumin; SOD, sphincter of Oddi dysfunction. 